\title{
Determinants of value transfer and contrast in simultaneous discriminations by pigeons
}

\author{
TRICIA S. CLEMENT and THOMAS R. ZENTALL \\ University of Kentucky, Lexington, Kentucky
}

\begin{abstract}
In a simultaneous discrimination involving a positive ( $\left.\mathrm{S}_{+}\right)$and a negative $\left(\mathrm{S}^{-}\right)$stimulus, positive value appears to transfer from the $S+$ to the $S-$. However, negative value does not appear to transfer from the $\mathrm{S}-$ to the $\mathrm{S}+$. Instead, when sufficient experience with the contingencies associated with responding to the $\mathrm{S}-$ is provided, it appears that the presence of the $\mathrm{S}-$ enhances the value of the $\mathrm{S}+$ (i.e., a contrast effect is found). The purpose of the present experiments was to further examine the influence of the $\mathrm{S}+$ on the $\mathrm{S}-$ in a simultaneous discrimination (between subjects in Experiment 1 and within subjects in Experiment 2). In both experiments, we found that under typical training conditions, given little direct experience with the value of the $S-$, value transfers from the $S+$ to the $S-$. If sufficient experience with the value of the $S-$ is provided, however, contrast between the $S+$ and the $S-$ can be demonstrated. Thus, in a simultaneous discrimination, value transfer from the $\mathrm{S}+$ to the $\mathrm{S}-\mathrm{de}-$ pends on the animal's having responded relatively little to the $S-$.
\end{abstract}

Fersen, Wynne, Delius, and Staddon (1991) proposed that in a simple simultaneous discrimination, positive value (similar to Hull's, 1943, notion of excitation) from the stimulus associated with reinforcement $(\mathrm{S}+$ ) can transfer to the stimulus not associated with reinforcement ( $\mathrm{S}-$ ), thus increasing the relative value of the $S-$. They also proposed that negative value (similar to Hull's notion of inhibition) can transfer from the $S-$ to the $S+$, thus decreasing the relative value of the $S+$. The mechanism by which such transfer occurs is similar to stimulus generalization, but it does not depend on the similarity between the $S_{+}$and the $S-$, as suggested by Spence (1937), but rather on the spatial and temporal proximity of the two simultaneously presented stimuli. Although it is reasonable to posit such transfer of value between $\mathrm{S}+$ and $\mathrm{S}-$, Fersen et al. provided no direct evidence in support of value transfer theory.

Zentall and Sherburne (1994) tested the hypothesis that positive value can transfer from the $S+$ to the $S-$ by using a procedure in which pigeons were trained on two simultaneous discriminations. In one simultaneous discrimination, responses to the $S+$ were always reinforced, whereas in the other discrimination, responses to the $\mathrm{S}+$ were reinforced only half of the time. In both discriminations, responses to the $S-$ were never reinforced. Thus, the two discriminations can be designated $\mathrm{A}_{100} \mathrm{~B}_{0}$ and $\mathrm{C}_{50} \mathrm{D}_{0}$ (where the subscripts indicate the percentage of the time that reinforcement was provided for responding to each of the stimuli). Following acquisition of these dis-

The research presented here was supported by Grants MH551 18 and MH59194 from the National Institute of Mental Health to T.R.Z. We thank Janice E. Weaver and Joann Feltus for their contribution to this research. Correspondence should be sent to T. R. Zentall, Department of Psychology, University of Kentucky, Lexington, KY 40506 (e-mail: zentall@pop.uky.edu). criminations, the pigeons were given a choice between the two negative stimuli (B and D). According to value transfer theory, A should have had more value to transfer to B than C should have had to transfer to D. In support of value transfer theory, Zentall and Sherburne found that animals trained in this manner consistently chose B over D. Subsequent research has validated this effect (e.g., Steirn, Weaver, \& Zentall, 1995; Zentall, Sherburne, Roper, \& Kraemer, 1996; Zentall, Weaver, \& Sherburne, 1996).

Recently, Clement, Weaver, Sherburne, and Zentall (1998) tested the second of Fersen et al.'s (1991) hypotheses, that negative value from the $\mathrm{S}$ - may transfer to its associated $\mathrm{S}+$, resulting in a decrease in the relative value of the S+. Clement et al. (1998) trained pigeons using a procedure similar to that used by Zentall and Sherburne (1994), but in this case, the values of the $S+$ stimuli were equated, whereas the values of the $\mathrm{S}$ - stimuli were varied $\left(A_{100} B_{0}\right.$ and $\left.C_{100} D_{50}\right)$. In a preliminary experiment, they found that the value of the $S$ - had little effect on the value of the $\mathrm{S}+$ with which it was presented. Clement et al. noted, however, that because the two simultaneous discriminations were acquired with very few errors, the pigeons had responded relatively little to the two $S-$ stimuli, and thus they may not have learned sufficiently about their differential value. To avoid this problem, Clement et al. (Experiment 1) gave pigeons direct experience responding to the two $\mathrm{S}-$ stimuli, in the form of single-stimulus exposures. The pigeons were trained on two simultaneous discriminations in which both $\mathrm{S}+$ stimuli were associated with reinforcement on $75 \%$ of the trials $(75 \%$ reinforcement was provided for correct responses to the $\mathrm{S}+$ stimuli to avoid possible ceiling effects on test trials that provided the pigeons with a choice between two $\mathrm{S}+$ stimuli). One $\mathrm{S}+$ was presented with an $\mathrm{S}-$ to which responses were never reinforced, and the other $\mathrm{S}+$ 
was presented with an $\mathrm{S}$ - to which responses were reinforced $25 \%$ of the time $\left(A_{75} B_{0}, C_{75} D_{25}\right)$. To ensure adequate experience with the contingencies associated with responding to the $S-$ stimuli, single-stimulus presentations of each $\mathrm{S}-$ were included during training $\left(\mathrm{B}_{0}\right.$, $\mathrm{D}_{25}$ ). On test trials, when the pigeons were given a choice between the two S+ stimuli, a consistent preference for A over $\mathrm{C}$ was found (i.e., a preference was found for the $\mathrm{S}+$ presented with the $\mathrm{S}-$ which was never reinforced). Thus, contrary to predictions by Fersen et al., it appears that negative value does not transfer from the $S-$ to the $S+$. Instead, the relative positive value of the $S+$ appears to depend on its degree of contrast with the $S-$ with which it was presented.

Clement et al. (1998, Experiment 2) also addressed the possibility that the contrast effect that they found was produced by the "hybrid" successive/simultaneous task used (i.e., single-stimulus presentations of the $S$ - stimuli were presented successively with the simultaneous discriminations). They trained one group of pigeons with the same discriminations used by Zentall and Sherburne (1994) but added single-stimulus presentations of the positive stimuli $\left(A_{100} B_{0}, C_{50} D_{0}, A_{100}, C_{50}\right)$. Following this training, the pigeons were given a choice between $\mathrm{B}$ and $D$ and positive value transfer was still found (B was preferred over D). Thus, it appeared that the hybrid nature of the task used to examine the effects of the $S-$ on the $\mathrm{S}+$ (i.e., single-stimulus presentations, during training, of the stimuli not used in test) could not account for the contrast found by Clement et al.

It is possible, however, that when there is little responding to one of the stimuli in a simultaneous discrimination, some of the value associated with the stimulus to which more responding has occurred transfers to (or is attributed to) the stimulus to which less responding has occurred. In the experiments in which the value of the Swas manipulated to determine its effect on the $S+$, however, the pigeons had responded extensively to both negative stimuli. It may be that when the actual value of the stimulus to which less responding had occurred can be assessed better, contrast will be found in both directions between the $\mathrm{S}+$ and the $\mathrm{S}$ - (i.e., manipulation of the value of the of the $\mathrm{S}+$ stimuli will result in contrast, as well).

If this argument is correct, in a simultaneous discrimination one should be able to demonstrate either negative contrast or the opposite, positive value transfer, depending on whether the contingencies associated with responding to the $\mathrm{S}-$ stimuli are well experienced or not.

\section{EXPERIMENT 1}

In Experiment 1, the positive transfer design used by Zentall and Sherburne (1994) was modified to allow additional responding to each of the $\mathrm{S}-$ stimuli. This was accomplished by including independent presentations of each $\mathrm{S}-$, in addition to the simultaneous discriminations. The pigeons received experience with two simultaneous discriminations. In one discrimination, the $\mathrm{S}-$ was pre- sented with an $\mathrm{S}+$ to which responses were reinforced on $100 \%$ of the trials, and in the other discrimination, the $S-$ was presented with an $\mathrm{S}+$ to which responses were reinforced on $50 \%$ of the trials. In addition, the pigeons also experienced single-stimulus presentations involving each of the $S-$ stimuli. To ensure that the pigeons continued to respond to the negative stimuli when they were presented alone, reinforcement was provided for responding on a small number $(12.5 \%)$ of the single-stimulus trials. For consistency, choice of the $\mathrm{S}-$ on simultaneous discrimination trials also was reinforced on $12.5 \%$ of the trials on which it was chosen.

If the relative absence of experience with the consequences of responding to the $S$ - stimuli was responsible for the value transfer found by Zentall and Sherburne (1994), contrast should be found in the present experiment. Alternatively, if additional experience with the consequences of $\mathrm{S}$ - responding afforded by independent presentations of the $\mathrm{S}$ - was not responsible for the positive contrast found by Clement et al. (1998), positive value transfer should occur in the present experiment.

\section{Method}

\section{Subjects}

The subjects were 8 experimentally naive White Carneaux pigeons (Columba livia) obtained from the Palmetto Pigeon Plant (Sumter, SC) as retired breeders. The pigeons were 5-8 years old; both male and female pigeons were used in the experiment. All of the pigeons were kept at $80 \%$ of their free-feeding body weights on a diet of Purina Pro Grains, provided primarily during experimental sessions. Each bird was housed individually in a cage located in a ventilated temperature-controlled colony room that was illuminated on a 12:12-h light:dark cycle. Water and grit were freely available in the home cage.

\section{Apparatus}

The pigeons were trained and tested in a BRS/LVE (Beltsville, MD) operant chamber measuring $37.5 \mathrm{~cm}$ high, $31 \mathrm{~cm}$ from the front panel to the back wall, and $35.5 \mathrm{~cm}$ from side to side. Three horizontally aligned rectangular $(3.5 \times 3.0 \mathrm{~cm})$ response keys were located on the front panel, $1 \mathrm{~cm}$ apart and $26.5 \mathrm{~cm}$ from the center of the keys to the floor of the chamber. Only the center and left response keys were used in the experiment. An inline projector mounted behind each response key could illuminate red $(\mathrm{R})$, yellow (Y), blue (B), and green (G) fields (Kodak Wratten Filter Nos. 26, 9,38 , and 60 , respectively). The bottom edge of a feeder aperture $(6.0 \times 5.5 \mathrm{~cm})$ was located $11.5 \mathrm{~cm}$ from the wire-mesh floor. Reinforcement consisted of 2-sec access to Purina Pro Grains from the feeder, which was lit whenever grain was available. An exhaust fan and white noise masked extraneous sounds. The events in the operant chamber were controlled by a microcomputer located in an adjacent room.

\section{Procedure}

Pretraining. All pigeons were trained to eat from the grain feeder and were then shaped by successive approximation to respond once (fixed ratio 1, FR 1) to each of the four stimuli (R, Y, G, B) located on each response key. The pigeons were required to complete one 24-trial, FR1 session (three presentations of each stimulus in each location, randomly determined). Following completion of the FR1 session, the response requirement was increased to five (FR5) for another 24-trial session. Simultaneous discrimination training began the following day. 
Discrimination training. The four hues $(R, Y, G, B)$ were assigned to two discriminations, counterbalanced over four subgroups (with 2 pigeons in each subgroup) such that each hue served equally often as A, B, C, and D. As in prior research, all discriminations were between red and yellow and between green and blue in order to reduce the possible effects of stimulus generalization on test trials when one hue from each discrimination would be presented together for the first time. On discrimination trials, five consecutive pecks to either stimulus constituted a choice that ended the trial and started the $10-\mathrm{sec}$ ITI. For the stimulus pair designated as AB, the fifth response to A was always reinforced, whereas the fifth response to $B$ was reinforced on only $12.5 \%$ of the trials $\left(A_{100} B_{12.5}\right)$. For the pair designated $C D$, the fifth response to $C$ was reinforced on a random half of the trials, whereas the fifth response to $D$ was reinforced on only $12.5 \%$ of the trials $\left(\mathrm{C}_{50} \mathrm{D}_{12.5}\right)$. Each session consisted of 128 trials. There were $32 \mathrm{AB}$ trials, $32 \mathrm{CD}$ trials, and 32 single-stimulus presentations each of $B$ and D. Each stimulus appeared equally often on the left and right response keys on both discrimination and single-stimulus trials. All pigeons were trained on this task to a performance criterion of $90 \%$ or better on both discriminations, for 2 consecutive days. After attainment of this criterion, half of the pigeons ( 1 from each subgroup) received test trials on the following day. The remaining pigeons received two additional training sessions (overtraining) before advancing to test. Half of the pigeons were given overtraining because it was thought that overtraining would increase the effects of direct experience with the consequences of responding to the $\mathrm{S}$-.

Test. During each of two test sessions, 16 test trials involving the BD pair were randomly intermixed among 112 training trials. The test stimuli appeared equally often on the two response keys. Responding to either stimulus was reinforced on a random $50 \%$ of the test trials. The design of Experiment 1 appears in Table 1.

\section{Results and Discussion}

\section{Acquisition}

The $\mathrm{A}_{100} \mathrm{~B}_{12.5}$ discrimination was acquired in a mean of 2.25 sessions, whereas the $\mathrm{C}_{50} \mathrm{D}_{12.5}$ discrimination was acquired in a mean of 2.37 sessions (including criterion sessions, but not overtraining sessions). The mean number of choices of B (presented with A) in training was 4.9 , and the mean number of choices of $D$ (presented with $C$ ) in training was 11.0. In addition, there was an average of 132 single-stimulus presentations each of $B$ and $\mathrm{D}$ during training. In all analyses, the .05 level of statistical significance was adopted.

Table 1

Design of Experiment 1

\begin{tabular}{cccc}
\multicolumn{4}{c}{ Discrimination Training } \\
\hline$\frac{\mathrm{A}_{100} \mathrm{~B}_{12.5}}{\mathrm{C}_{50} \mathrm{D}_{12.5}}$ & Test & $\mathrm{B}_{12.5}$ & $\mathrm{D}_{12.5}$
\end{tabular}

$B$ versus D

Note-Training involved 32 trials per session involving each simultaneous discrimination and each single-stimulus presentation. Testing involved two sessions consisting of $16 \mathrm{BD}$ trials interspersed among 28 trials involving each of the training trial types. Subscripts refer to the probability of reinforcement associated with responding to each of the stimuli.

\section{Test}

Overall, the pigeons preferred D over B on $76.47 \%$ of the test trials. An analysis of variance (ANOVA) performed on these preference scores, with overtraining as a factor, indicated that the preference for D over B was significantly above chance $[F(1,6)=7.75]$ and did not vary as a function of overtraining $(F<1)$. Preference for D over $B$ indicates a finding of contrast between the $\mathrm{S}+$ and the $\mathrm{S}-$ in a simultaneous discrimination, because, in training, the value associated with $\mathrm{B}$ and $\mathrm{D}$ should have been the same. Instead, the pigeons showed a preference for the $S$ - that in training was presented with the lesser valued $\mathrm{S}+\left(\mathrm{C}_{50}\right)$ over the $\mathrm{S}-$ that in training was presented with the greater valued $\mathrm{S}+\left(\mathrm{A}_{100}\right)$. Thus, the $\mathrm{S}-$ associated with the $S_{50}$ appeared relatively better than the $S-$ associated with the $S_{100}$.

The implications of this finding are that in a simultaneous discrimination the effect of an $\mathrm{S}+$ on its $\mathrm{S}-$ will depend on the degree to which the actual value of the $S-$ is known. If there has been little direct experience with the $\mathrm{S}-$ (as is often the case with a relatively easy simple simultaneous discrimination) value appears to transfer from the $\mathrm{S}+$ to the $\mathrm{S}-$, enhancing the value of the $\mathrm{S}-$. If, however, the value of the $S$ - has been sufficiently experienced, the value of the $S-$ will be judged relative to (or in contrast to) the value of the $\mathrm{S}+$ with which it was presented.

\section{EXPERIMENT 2}

Experiment 1 established that although in a typical simultaneous discrimination, value generally transfers from the $\mathrm{S}+$ to the $\mathrm{S}-$, if additional experience is given with the contingencies associated with $S$ - responding, contrast between the $S+$ and the $S-$ might result. The purpose of Experiment 2 was to determine whether we could demonstrate both value transfer and contrast within the same subject-that is, whether we could first demonstrate positive value transfer using the traditional discrimination design, but with single-stimulus presentations of the positive stimuli, and second, whether we could reverse that preference by replacing the single-stimulus presentations of the positive stimuli with single-stimulus presentations of the negative stimuli.

In Experiment 2, the pigeons were trained with two simultaneous discriminations, both involving an $\mathrm{S}-$ associated with reinforcement on $12.5 \%$ of the trials on which the $S-$ was chosen. Choice of the $S+$ associated with one $S-$ was reinforced $100 \%$ of the time, whereas choice of the $\mathrm{S}+$ associated with the other $\mathrm{S}$ - was reinforced $50 \%$ of the time $\left(\mathrm{A}_{100} \mathrm{~B}_{12.5}, \mathrm{C}_{50} \mathrm{D}_{12.5}\right)$. In Phase 1, discrimination training was accompanied by independent presentations of each $\mathrm{S}+$ (as a control for the number of singlestimulus trials presented during both phases; see Clement et al., 1998, Experiment 2). If positive value transfers 
from the $S+$ to the $S$ - only when the direct value of the $\mathrm{S}-$ stimulus is not well experienced, then following this training, when given a choice between $\mathrm{B}$ and $\mathrm{D}$, the pigeons should prefer B over D. However, if in Phase 2, independent presentations of each of the two $S-$ stimuli replace the independent presentations of each of the two $\mathrm{S}+$ stimuli, the pigeons should reverse their preference (i.e., they should then prefer D over B).

\section{Method}

\section{Subjects}

The subjects were 16 experimentally naive White Carneaux pigeons similar to those used in Experiment 1. The subjects were housed and maintained as in Experiment 1.

\section{Apparatus}

The apparatus and stimuli used in Experiment 2 were the same as in Experiment 1.

\section{Procedure}

Pretraining. Pretraining was the same as in Experiment 1.

Phase 1. Training and test were the same as in Experiment 1, with the exception that there were single-stimulus presentations of the $A$ and $C$ stimuli $\left(A_{100} B_{12.5}, C_{50} D_{12.5}, A_{100}, C_{50}\right)$, rather than the $B$ and $D$ stimuli experienced in Experiment 1 . Because in Experiment 1 there was little effect of overtraining, all of the pigeons were tested after 2 consecutive days at $90 \%$ or better on both discriminations.

Test 1. As in Experiment 1, two test sessions followed training and each test session contained $16 \mathrm{BD}$ test trials in addition to 112 training trials.

Phase 2. Following the first test, the pigeons were returned to the discrimination training procedure, but independent presentations of the $B$ and $D$ stimuli replaced the independent presentations of the $A$ and $C$ stimuli $\left(A_{100} B_{12.5}, C_{50} D_{12.5}, B_{12.5}, D_{12.5}\right)$. Because all pigeons had previously acquired each discrimination to a high level of accuracy, no performance-based criterion was adopted prior to test. Instead, to control for total single-stimulus experience (with the $\mathrm{S}+$ stimuli in Phase 1 and the $\mathrm{S}-$ stimuli in Phase 2), each pigeon received the same number of training sessions in Phase 2 as in Phase 1.

Test 2. Following Phase 2 training, the pigeons received two test sessions, each involving $16 \mathrm{BD}$ test trials, as in Experiment 1 . The design of Experiment 2 appears in Table 2.

Table 2

Design of Experiment 2

\begin{tabular}{|c|c|c|c|}
\hline \multicolumn{4}{|c|}{ Phase 1: Discrimination Training } \\
\hline Simultaneo & criminations & Single- & raining \\
\hline $\mathrm{A}_{100} \mathrm{~B}_{12.5}$ & $\begin{array}{r}\mathrm{C}_{50} \mathrm{D}_{12.5} \\
\mathrm{~B}\end{array}$ & $\begin{array}{l}A_{100} \\
D\end{array}$ & $\mathrm{C}_{50}$ \\
\hline
\end{tabular}

Phase 2: Discrimination Training

\begin{tabular}{|c|c|c|c|}
\hline Simultaneo & criminations & Single & Training \\
\hline $\mathrm{A}_{100} \mathrm{~B}_{12.5}$ & $\begin{array}{r}\mathrm{C}_{50} \mathrm{D}_{12.5} \\
\mathrm{~B}\end{array}$ & $\begin{array}{l}\mathrm{B}_{12.5} \\
\mathrm{D}\end{array}$ & $\overline{D_{12.5}}$ \\
\hline
\end{tabular}

Note-Training Phases 1 and 2 involved 32 trials per session involving each simultaneous discrimination and each single-stimulus presentation. Testing involved two sessions consisting of $16 \mathrm{BD}$ trials interspersed among 28 trials involving each of the training trial types. Subscripts refer to the probability of reinforcement associated with responding to each of the stimuli.

\section{Results and Discussion}

\section{Phase 1}

The $\mathrm{A}_{100} \mathrm{~B}_{12.5}$ discrimination was acquired in a mean of 2.88 sessions, whereas the $\mathrm{C}_{50} \mathrm{D}_{12.5}$ discrimination was acquired in a mean of 3.31 sessions (including criterion sessions). This difference was not statistically significant $(F<1)$. The mean number of choices of B (presented with $A$ ) in training was 18.9 and the mean number of choices of D (presented with C) in training was 24.1.

\section{Test 1}

After single-stimulus training with the $\mathrm{S}+$ stimuli, pigeons chose B (the stimulus that had been presented with the more valued positive stimulus, A) over D (the stimulus that had been presented with the less valued positive stimulus, C) on $84.37 \%$ of the test trials. An ANOVA performed on the preference scores indicated that the preference for B over D was significantly greater than chance $[F(1,14)=5.33]$. Thus, the pigeons that responded very little to the $\mathrm{S}-$ stimuli, but that experienced an amount of single-stimulus training similar to that experienced by the pigeons in Experiment 1, transferred positive value from the $\mathrm{S}+$ to the $\mathrm{S}-$.

\section{Phase 2}

There were virtually no responses made to either B $(M=0.38)$ or $\mathrm{D}(M=0.25)$ during retraining when these stimuli were presented in simultaneous discriminations with $A$ and $C$, respectively. There was an average of 140 single-stimulus presentations each of $\mathrm{B}$ and $\mathrm{D}$ during Phase 2.

\section{Test 2}

Consistent with the results of Experiment 1, when single-stimulus presentations of the $\mathrm{S}-$ stimuli replaced the single-stimulus presentations of the $\mathrm{S}+$ stimuli, the pigeons reversed their prior preference and chose $D$ over $B$ on $78.64 \%$ of the test trials. An ANOVA performed on these preference scores indicated that the preference for $D$ over $\mathrm{B}$ was significantly above chance $[F(1,14)=14.63]$. This clear reversal in preference between the two tests from the $\mathrm{S}-$ associated with the more valued $\mathrm{S}+$ to the $\mathrm{S}-$ associated with the less valued $\mathrm{S}+$ can only be attributed to the direct experience with the $\mathrm{S}-$ stimuli as single stimuli during Phase 2.

\section{GENERAL DISCUSSION}

There is now considerable evidence that in a simultaneous discrimination, the value of the $\mathrm{S}+$ can affect the value of the $\mathrm{S}$ - with which it is presented. Previous research suggests that in a typical simultaneous discrimination some of the value of the $S+$ transfers to the $S-$, a result consistent with value transfer theory (Fersen et al., 1991). If two S- stimuli, of the same nominal value, are each associated with a different $\mathrm{S}+$ stimulus, one having more value than the other, on test trials pigeons prefer the 
$\mathrm{S}$ - that in training was presented with the $\mathrm{S}+$ of greater value (see, e.g., Zentall \& Sherburne, 1994).

However, the value of the $S$ - does not appear to have a corresponding effect on its associated $\mathrm{S}+$. Thus, if two $\mathrm{S}+$ stimuli, of the same nominal value, are each associated with a different $\mathrm{S}$ - stimulus, one having more value than the other, and the pigeons have adequate experience responding to both $\mathrm{S}-$ stimuli by themselves, on test trials, pigeons prefer the $S+$ that was presented with the $S-$ of lesser value (i.e., contrast rather than value transfer is found; Clement et al., 1998).

The present results extend those earlier findings to the effect of the $\mathrm{S}+$ on the $\mathrm{S}-$. Although there is evidence for value transfer from the $S+$ to the $S-$ when there has been relatively little experience with the contingencies associated with responding to the $S-$ stimuli (Phase 1 of Experiment 2 of the present research), when adequate experience is given with those contingencies, there is evidence for contrast (Experiment 1 and Phase 2 of Experiment 2 of the present study).

The contrast effects found in the present study do not appear to result from the hybrid nature of the procedure used to provide direct experience with the value of the $\mathrm{S}-$ stimuli, per se. If single-stimulus presentations of the untested stimuli were sufficient to produce contrast between the S+ and S-, contrast (rather than value transfer) should have been found when the $S+$ stimuli were presented as single stimuli (in Phase 1 of Experiment 2 of the present study). Furthermore, the effects found in the present experiments cannot be attributed to simple successive contrast (similar to behavioral contrast; see Reynolds, 1961), because if the pigeons treated the task as a fourcomponent successive discrimination (or multiple schedule), there is no reason for the two $\mathrm{S}+$ stimuli to affect the two S- stimuli differentially. Clearly, the mechanism for the differential effects of the two S+ stimuli on the two $S-$ stimuli must result directly from the appearance of the two stimuli in a simultaneous discrimination.

Thus, it appears that in a typical simultaneous discrimination with minimal responding to the negative stimuli (in Experiment 2, Phase 1, the pigeons chose each of the $\mathrm{S}-$ stimuli about 20 times before reaching criterion), value transfers from the $S+$ to the $S-$, but the $S-$ has little effect on the $S+$. When pigeons are given direct experience with the $S-$, in the form of single-stimulus training (in Experiments 1 and 2, about 140 presentations), however, not only does the lesser valued $\mathbf{S}-$ enhance the relative value of the $\mathrm{S}+$ with which it appeared (Clement et al., 1998), but, as demonstrated in the present experiments, the more valued $\mathrm{S}+$ diminishes the relative value of the $S-$ with which it appeared. Thus, value transfers from the $\mathrm{S}+$ to the $\mathrm{S}-$ only when there has been minimal experience with the consequences of responding to the $\mathrm{S}-$.

Although the present findings were obtained with simultaneous discriminations, they also might be related to other reported phenomena. For example, Terrace (1963) found that if limited exposure was provided to the
$\mathrm{S}$ - in a successive $\mathrm{S}+\mathrm{S}-$ discrimination by initially presenting the $\mathrm{S}$ - very briefly and under very dim illumination and then gradually increasing its intensity as well as its duration, pigeons could learn to respond to the $\mathrm{S}+$ and not to the $S$ - without ever having responded to the $\mathrm{S}-$. Furthermore, Terrace reported that when pigeons acquire such a discrimination without errors, the $S-$ does not become aversive, does not develop inhibitory properties, and does not result in positive behavioral contrast to the $\mathrm{S}+$. The present results are quite consistent with Terrace's finding. In the simultaneous discrimination, although some errors were made to the $\mathrm{S}-$, the number of errors was very few when compared with those for a successive discrimination. In the typical simultaneous discrimination, not only does the S - not take on inhibitory properties, it actually appears to gain excitatory properties, perhaps because of its temporal and spatial proximity to the S+.

The typical simultaneous discrimination may also provide a useful model for potentiation, a paradoxical phenomenon that has been reported in the conditioning literature in which conditioning of a weakly conditionable stimulus, such as an odor to illness, can be enhanced by combining the odor with a strongly conditionable stimulus, such as a taste to illness (Rusiniak, Hankins, Garcia, \& Brett, 1979). The reason that potentiation is considered paradoxical is that generally the pairing of a weakly conditionable stimulus with a strongly conditionable stimulus results in weaker conditioning to the weakly conditionable stimulus. This is a well-established phenomenon known as overshadowing (Kamin, 1969).

What may make simultaneous discrimination learning analogous to potentiation are three possibly important characteristics of potentiation that also appear to be present in the acquisition of a simultaneous discrimination. First, the pairing of the weakly conditionable stimulus, the odor, with illness is analogous to the poor association of the $S-$ with food. In the case of the $S-$, this poor association occurs because responses to the $S-$ are explicitly not reinforced. Second, it is likely that organisms are predisposed to associate odors with simultaneously presented tastes. Odors and tastes go together naturally. Similarly, in a simultaneous discrimination, the simultaneous presentation of S+ and S- (generally stimuli coming from the same stimulus dimension in close spatial proximity to each other) may make it likely that they too will be associated. And finally, because organisms appear to be predisposed to associate taste with illness, taste appears to be a particularly powerful conditioned stimulus in the context of illness. Similarly, the immediate reinforcement of responses to the $\mathrm{S}+$ means that it is likely to become a strongly excitatory stimulus.

Zentall, Sherburne, et al. (1996) have suggested that the pathway by which value transfers from the $S+$ to the $\mathrm{S}-$ may be higher order conditioning. Once a discrimination has been learned, the presence of the S- (to which responses are no longer made) becomes a signal for the presence of the S+. Zentall, Sherburne, et al. found 
evidence for such a pathway by first training a simultaneous discrimination and then devaluing the $\mathrm{S}+$ by extinguishing responding to it in the absence of the $S-$. Zentall et al. found that choice of the $\mathrm{S}-$ was greatly diminished following the devaluation of the S+. But Zentall et al. also found evidence for direct associations between the $S+$ and the $S-$ in a simultaneous discrimination, what has been referred to as within-event or configural learning (Pearce, 1987). Thus, the mere presentation of the $S+$ (without devaluation) in the absence of the $S-$ will reduce the transfer of value from the $S+$ to the $S-$.

This model of potentiation suggests that overshadowing will tend to occur when the first two characteristics of potentiation (and especially the second) are not met. First, the less salient stimulus-for example-a tone, should be (by itself) readily associated with food, which appears to be true (Kamin, 1969). And second, unlike taste and odor, which are easily associated when presented together, the tone is not so readily associated with the more salient stimulus - for example, a light. Therefore, unlike the special case of taste and odor, the pathway from the less salient tone through the more salient light to food is not any easier to make than the direct pathway from tone to food, and thus the two stimuli have to share the direct pathway and one may overshadow the other.

Therefore, in both simultaneous discrimination learning and potentiation, but not overshadowing, one stimulus is not easily associated with reinforcement (odor and $\mathrm{S}-$ ), but it is easily associated with another stimulus (taste and $\mathrm{S}+$ ) that in turn is easily associated with reinforcement. If one carries the analogy one step further, it leads to an interesting prediction. If direct experience with the $S$ - (through single-stimulus training) enhances the value of the $\mathrm{S}+$ with which it was presented, is it also true that explicit nonreinforced experience with the overshadowed stimulus (e.g., the tone) would enhance conditioning to the light (relative to a light-alone-conditioning control group)? Whether this discrimination learning analogy will prove helpful to an understanding of potentiation and its relation to overshadowing is not clear, but even if it merely encourages us to see these phenomena from a different perspective, it will have served a useful purpose.

\section{REFERENCES}

Clement, T. S., Weaver, J. E., Sherburne, L. M., \& Zentall, T. R. (1998). Effect of the $S-$ on the $S+$ in a simultaneous discrimination: Negative value transfer or contrast. Quarterly Journal of Experimental Psychology, 51B, 363-378.

Fersen, L. von, Wynne, C. D. L., Delius, J. D., \& Staddon, J. E. R. (1991). Transitive inference formation in pigeons. Journal of Experimental Psychology: Animal Behavior Processes, 17, 334-341.

Hull, C. L. (1943). Principles of behavior. New York: AppletonCentury-Crofts.

KAMIN, L. J. (1969). Predictability, surprise, attention, and conditioning. In B. A. Campbell \& R. M. Church (Eds.), Punishment and aversive behavior (pp. 279-296). New York: Appleton-Century-Crofts.

Pearce, J. M. (1987). A model for stimulus generalization in Pavlovian conditioning. Psychological Review, 94, 61-73.

REYNOLDS, G. S. (1961). An analysis of interactions in a multiple schedule. Journal of the Experimental Analysis of Behavior, 4, 107-117.

Rusiniak, K. W., Hankins, W. G., Garcia, J., \& BretT, C. P. (1979). Flavor-illness aversions: Potentiation of odor by taste in rats. Behavioral \& Neural Biology, 25, 1-17.

SPENCE, K. W. (1937). The differential response in animals to stimuli varying within a single dimension. Psychological Review, 44, 430-444.

Steirn, J. N., Weaver, J. E., \& ZenTall, T. R. (1995). Transitive inference in pigeons: Simplified procedures and a test of value transfer theory. Animal Learning \& Behavior, 23, 76-82.

Terrace, H. S. (1963). Errorless transfer of a discrimination across two continua. Journal of the Experimental Analysis of Behavior, 6, 223-232.

Zentall, T. R., \& Sherburne, L. M. (1994). Transfer of value from S+ to $\mathrm{S}-$ in a simultaneous discrimination. Journal of Experimental $P$ sychology: Animal Behavior Processes, 20, 176-183.

Zentall, T. R., Sherburne, L. M., Roper, K. L., \& Kraemer, P. J. (1996). Value transfer in a simultaneous discrimination appears to result from within-event Pavlovian conditioning. Journal of Experimental Psychology: Animal Behavior Processes, 22, 68-75.

Zentall, T. R., Weaver, J. E., \& Sherburne, L. M. (1996). Value transfer in concurrent-schedule discriminations by pigeons. Animal Learning \& Behavior, 24, 401-409.

(Manuscript received September 30, 1999; revision accepted for publication January 18,2000 .) 